\title{
ETNOGRAFÍAS MULTISITUADAS Y MULTIFOCALIZADAS: Cuando es «el campo» el que te lleva. Siguiendo a los sikhs y descu- briendo a los gora sikhs
}

\author{
Sandra SANTOS FRAILE \\ Universidad Pablo de Olavide \\ sandra.santos.fraile@gmail.com
}

\begin{abstract}
MULTISITED AND MULTIFOCUSED ETHNOGRAPHIES: When it is fieldwork that guides you. Following the Sikhs and discovering the Gora Sikhs
\end{abstract}

Resumen: El objetivo de este artículo es plantear la necesidad o la inevitabilidad de la reformulación constante y contextual de la metodología antropológica. También, de forma más específica, del planteamiento de los proyectos de investigación en directa relación con el mundo global y las interconexiones transnacionales que este inexorablemente implica como elemento necesario para el logro de etnografías exitosas en la actualidad. Para ello expongo algunas dificultades y modificaciones necesarias que tuve que llevar a cabo como parte del proceso del trabajo de investigación para mi tesis doctoral. Así, la pretensión de este texto es, por un lado, visibilizar el hecho de que es el propio trabajo de campo quien te lleva a redefinir continuamente no sólo la estrategia previamente planificada para la realización de la etnografía, sino también quiénes serán los sujetos de estudio necesarios para la viabilidad del trabajo etnográfico. Por otro lado, reivindicar la necesidad de normalizar las etnografías multisituadas y multifocalizadas para dar cuenta de la realidad actual de nuestros sujetos de estudio, que ya no pueden delimitarse a un grupo cerrado o a un espacio-territorio acotado. En este sentido, reflexionar sobre las formas o modos de llevar a cabo este tipo de etnografías diseminadas, se torna condición necesaria para poder describir la realidad de la que hoy forman parte nuestros grupos o temáticas de estudio.

Abstract: The aim of this article is to show the necessity or the inevitability of the constant and contextual reformulation of anthropological methodology. In addition, and more specifically, it is to consider the importance of setting out our research projects in direct relation to the global world and the transnational interconnections that it inexorably implies, this consideration currently being a necessary element for the achievement of successful ethnographies. In order to achieve this, I will present some difficulties and the modifications that I had to make as part of the research process for my doctoral thesis. The text will show that is fieldwork itself that continually guides us and makes us redefine not only the previously planned strategy for carrying out ethnography, but also who will be the study subjects necessary for the viability of ethnographic work. I also intend to highlight the need to normalize multisited and multifocused ethnographies in order to account for the current reality of our study subjects, who can no longer be limited to a closed group or a bounded space or territory. Reflecting on the ways to carry out this type of scattered ethnography becomes a necessary condition for describing the reality of which our study groups or topics are a part.

Palabras clave: Etnografía multi-situada y multi-focalizada. Transnacionalismos y globalización. Trabajo de campo. Sikhs. Gora Sikhs

Multi-sited and multi-focused ethnography. Transnacionationalisms and globalization. Fieldwork. Sikhs. Gora Sikhs 


\section{Introducción}

Este artículo trata de reflexionar sobre la necesidad o la inevitabilidad de la reformulación constante y contextual de la metodología antropológica y, de forma más específica, del planteamiento de los proyectos de investigación para la consecución de etnografías exitosas en el mundo de hoy. Para ello, voy a referir al trabajo de mi de mi tesis doctoral (SantosFraile, 2016) cuyo objetivo consistía en analizar los cambios y transformaciones en el cuerpo y la corporalidad tras el proceso migratorio de la comunidad sikh ${ }^{1}$ como forma de adaptación a un nuevo contexto, en este caso, la ciudad de Barcelona. Comenzaré planteando algunas cuestiones identitarias que caracterizan a este grupo en tanto que comunidad, donde las relaciones transnacionales son un elemento fundamental y cómo esto me llevó a la necesidad de realizar etnografía multisituada. Más adelante expongo cómo sería precisamente esta metodología de trabajo la que me permitiría comparar las diferentes estrategias y alianzas que los sikhs desarrollan en los diferentes contextos de llegada y cómo esto me llevaría además a conocer a los gora sikhs y saber de su existencia en nuestro país. Finalmente, y de forma un tanto somera por los límites que un trabajo como este implica, hago un acercamiento a los principios ideológicos y prácticas cotidianas que tienen en común los gora sikhs con los sikhs venidos de la India, pero especialmente a las diferencias y distancias que (re)crean para marcar su propia idiosincrasia y su distinción un grupo con respecto al otro.

Tan pronto como dio comienzo la investigación para la tesis pude constatar que era del todo imposible conocer sobre los sikhs -y de forma más específica sobre las transformaciones en su corporalidad, aunque no solo- sin considerar cuestiones relativas a su construcción identitaria. Por un lado, los sikhs de origen hindú se autodefinen como una comunidad religiosa global. Y, de hecho, para poder definir a los sikhs hay que considerar su pertenencia a una religión particular (el sikhismo) pero también a un origen o ascendencia común (el Panyab) y a un lenguaje compartido (el panyabi). Asimismo, además de por una cultura compartida, los sikhs se vinculan por un sentimiento de hermandad a niveles transnacionales y globales (op.cit.), es decir, que trasciende, de lejos, los límites locales o comunales. Cabe señalar aquí que Según Matringe (2008: 263), más de un millón y medio de sikhs están establecidos en el extranjero, lo que según el autor correspondería a la proporción más elevada de cualquier comunidad religiosa de la India. Este significativo establecimiento fuera de su tierra natal respondería a una importante tradición emigratoria panyabi que se remonta más allá que la propia religion sikh (Cole \& Singh Sambhi, 2006: 182). Ya se conocen movimientos de comerciantes sikhs en el siglo XVI en Afganistán, Persia y Ceilán (Matringe, 2008: 263). Asimismo, McLeod (2004: 242) explica cómo ya siglos antes de la ocupación británica de la India multitud de sikhs se habían establecido en otras zonas del país como consecuencia de la creación de redes comerciales, sentando las bases de lo que más tarde serían los patrones migratorios que según este autor continuarían hasta la actualidad. A pesar de ello, parece que la anexión británica del Panyab sería el primer hito histórico en este sentido que haría que a partir de 1849 muchos panyabis se marcharan a otros continentes.

\footnotetext{
1 Pese a que a nivel popular y en la Real Academia Española en su edición más reciente se utilizan el vocablo sij y su derivado sijismo como transliteración del término sikh, tal y como expongo y justifico en mi tesis doctoral (Santos-Fraile, 2016) esta adaptación al español es bastante cuestionable ya que se basaría en un error de interpretación del valor fonético de la secuencia consonántica " $k h$ ". Dado que no es este lugar para alargarme sobre esta cuestión, sólo señalar que he optado por mantener el uso del vocablo sikh y de su derivado sikhismo, de igual modo que se hace en el mundo académico anglosajón y francófono, donde tampoco existirían el sonido " $k h$ " como tal. Para más información al respecto se pueden consultar fuentes de referencia como los diccionarios sánscrito-inglés e hindi-inglés clásicos de Monier-Williams (1996), el de McGregor (2004) o el diccionario de sánscrito-catalán de Pujol (2005). Otro autor que ha mantenido y defiende el uso de los términos sikh y sikhismo es Agustín Pániker (2007).
} 
Tanto es así que en la actualidad más de un tercio de la población del centro del Panyab se encuentra emigrada al extranjero, encontrándose hoy importantes comunidades panyabis en África Oriental, Sudeste Asiático, Canadá, Estados Unidos y Europa Occidental. A pesar de que las políticas restrictivas de entrada de estos migrantes a los distintos países van a condicionar fuertemente sus lugares de elección, los sikhs han mostrado y siguen mostrando una importante preferencia por los países de habla inglesa, donde siguen siendo Estados Unidos, Canadá y Gran Bretaña sus destinos prioritarios, considerados destinos de prestigio, y a los que se llega también desde África Oriental y África del Sur así como desde el Sudeste Asiático y Fiyi. Aunque otros países europeos, al igual que Australia, Japón y algunos países del Golfo Pérsico también han visto crecer su población sikh. A pesar de esta gran dispersión, podríamos definirlos como una comunidad imaginada, (Anderson, 2000) a escala global, saturada de relaciones políticas e identitarias que en muchos casos están vinculadas a diferentes procesos de definición étnica y religiosa en los distintos contextos. Más aún, esta hermandad estaría directamente relacionada con una búsqueda del reconocimiento - aunque en muchos casos también con la aspiración a la independencia de la India y la consiguiente conformación de un estado sikh soberano. No obstante, y a pesar de ser una comunidad religiosa global más o menos cohesionada, existen divisiones internas (ya que coexisten diferentes subgrupos de adscripción o pertenencia definidos como sectas ${ }^{2}$ ), en muchos casos relacionados con el sistema de castas.

En cualquier caso, lo religioso y su práctica son elementos fundamentales en la conformación de los sikhs, tanto como individuos así como miembros de la comunidad, de tal modo que sus prácticas y creencias afectan y organizan muchos aspectos de su vida cotidiana. De hecho, existe un código de conducta, el Rehat Maryada que regula y organiza muchos aspectos y prácticas de su vida cotidiana. Luego ser un o una sikh, va más allá de restringirse a una mera definición de los individuos por sus creencias; un o una sikh -dentro de la diversidad interna que, como decía más arriba, entre ellos existe- se definen también por determinada filosofía y modos de vida que los y las caracterizan. Y en los fundamentos de esta ética, como ya señalara Nikky-Guninder Kaur Singh (2004: 8-9), los sikhs se concentran en vivir su fe, donde la hermandad, la comunidad y la familia -como planteamientos de base- están en el centro del sikhismo, considerando a los otros y otras sikhs como hermanos y hermanas.

\section{Los sikhs de Barcelona y sus conexiones transnacionales}

Conocer y comprender cómo es, se organiza, gestiona sus prácticas y, en definitiva, produce y reproduce su cultura la comunidad sikh de Barcelona, tal y como se hizo evidente desde el comienzo del trabajo de campo, no puede lograrse si no es en relación con otros y otras sikhs que viven en la India o que se han establecido en diferentes lugares de la diáspora. La vinculación transnacional de la comunidad sikh de Barcelona, de hecho, se pone continuamente de manifiesto desde las relaciones y la solidaridad existentes en la comunidad, que trascienden los límites de lo local. Así, los sikhs mantienen frecuentes contactos con la familia extensa y el lugar de origen, no solamente a través del teléfono e Internet, sino también enviando remesas regulares que ayudan a mantener, incrementar o mejorar las propiedades y la estructura familiar, pero también para la creación o mejora de otros templos y espacios promocionados por los sikhs, como pueden ser colegios y otros centros de formación sikhs, residencias de ancianos o centros sanitarios -a saber, contribu-

2 Sigo aquí la categorización de secta de Joan Prat (2007: 63) quien la define como "un grupo religioso que defiende un sistema de creencias, nuevo, minoritario y extraño respecto de la fe (o fes) mayoritaria en la que el grueso de la sociedad ha sido educado y socializado". De modo que en nuestro caso los diferentes subgrupos o sectas dentro del sikhismo serían considerados como grupos minoritarios desviados respecto de la ideología hegemónica, mayoritaria y considerada hoy como ortodoxa. 
ciones filantrópicas como señala Portes (2007: 22) que tienden a realizar las organizaciones de migrantes transnacionales para ayudar a las comunidades locales (de origen), aunque en nuestro caso se dan también a nivel individual y hacia otras comunidades sikhs migradas-. Asimismo, desde Barcelona, se mantienen frecuentes contactos con la comunidad a nivel internacional con la que se colabora económica y éticamente, y de la que se está continuamente informado, ya sea a través de las versiones on-line de los periódicos panyabíes, los programas internacionales que visionan a través de las antenas parabólicas y los contactos frecuentes con los familiares y amigos que permanecen en la India como con aquellos otros miembros que emigraron a otros lugares. Frecuentes visitas a la tierra natal así como a otras comunidades de la diáspora es otro recurso habitual para mantener fuertes vínculos transnacionales. Los frecuentes contactos y las importantes conexiones que mantienen vienen, de este modo, tanto por la constante comunicación a través de las nuevas tecnologías, como por las visitas que con gran frecuencia se hacen unos a otros (a veces incluso entre desconocidos pero que tienen relaciones con personas en común o simplemente provienen del mismo pueblo) y por la participación colectiva y transnacional en la celebración de grandes eventos o festividades sikhs en distintos países. Especialmente cuando importantes eventos acontecen, como fue el gran langar ${ }^{3}$ que se preparó con ocasión del Forum Universal de las Culturas en 2004 en Barcelona, organizado por sikhs venidos de Birmingham y Canadá debido a la aún precaria organización de la comunidad sikh de Barcelona. Un ejemplo más reciente fue el incidente producido el 24 de mayo de 2009 en Viena cuando algunos sikhs pertenecientes a lo que hoy se considera la ortodoxia dispararon a dos líderes espirituales de la comunidad ravidassia ${ }^{4}$ considerados como herejes por los primeros ${ }^{5}$. Como consecuencia de este hecho, muchos seguidores se movilizaron a nivel internacional y la violencia se desató en el Panyab, donde el gobierno tuvo que declarar el toque de queda y enviar al ejército para restaurar el orden.

Asimismo, existe una especie de organización virtual y supranacional que de forma constante promueve y gestiona información manteniendo a los sikhs de los distintos países en relación, y donde la comunidad sikh de Barcelona participa activamente. Se utiliza para ello internet (a través páginas webs, listas de mailing, cuentas de youtube, etc.) ${ }^{6}$ como un recurso fundamental, pero no único. Así, por ejemplo, existe un registro oficial con sede en el Panyab con información y contacto de todos los gurdwaras ${ }^{7}$, especialmente los que están en la diáspora. En este se guarda una relación de cada nuevo templo que se abre, de tal forma que mantienen y ofrecen información constante sobre el número de gurdwaras fuera de la India ${ }^{8}$ y su ubicación, y donde aparecen registrados algunos de los templos ubicados en

3 Comida comunitaria y gratuita que se ofrece a cualquier persona en todos los gurdwaras o con objeto de alguna celebración.

4 Secta o sub-grupo religioso que se caracterizada por ser seguidores de Ravidass, considerado por éstos como gurú, pero por los sikhs más ortodoxos como bhagat, es decir, líder espiritual, pero no gurú. Este grupo se caracteriza además por ser principalmente de casta baja, como lo fue el propio Ravidass.

5 Que pertenecerían al grupo que hoy se considera como ortodoxo y controla las instancias de poder en la actualidad sikh.

6 Ejemplos de esto podemos encontrar en dos páginas webs internacionales en las que se informa y se solicita ayuda para cubrir las necesidades de sikhs en la diáspora, en este caso concreto en Valencia, donde tenían serias dificultades económicas para cubrir las carencias de un gurdwara. En la primera de las webs se explicita además cómo sikhs particulares desde distintas partes del globo -alguna tan alejada como Corea del Sur-, así como comunidades como la de Coventry, han aportado dinero para suplir estas necesidades. Ver: http://www.unitedsikhs. org/PressReleases/COMVCE-14-10-2006-00.htm [Última visita: 19/10/2017] y http://www.sevatothemax. com/blog/2006/11/12/gurdwara-sri-guru-singh-sabha-coventry-donates-to-help-spanish-sikhs/ [Última visita: 24/11/2008].

7 Nombre que reciben los templos sikhs, aunque también aquel espacio donde se encuentre su texto sagrado, el Gurú Granth Sahib.

8 Este registro permite asimismo el control financiero de los templos desde el Panyab, así como la obtención de 
el Estado español -aunque por el momento no todos-. La utilización de antenas parabólicas para ver la televisión vía satélite es un recurso informativo potente y muy utilizado al permitir la producción y emisión de programas realizados en los distintos países y que luego serán retransmitidos por toda una serie de canales panyabíes. Así como a Barcelona en distintas ocasiones han venido reporteros desde Londres y otras zonas de Gran Bretaña para grabar en los templos o durante alguna celebración, también hay corresponsales en Barcelona que se encargan de publicar y transmitir para medios informativos de la comunidad a nivel global. También la radio ayuda a que fluya la información gracias a programas producidos por los mismos panyabíes, tanto a nivel local como internacional ${ }^{9}$. Es, asimismo, que siempre que es posible los matrimonios se acuerdan a nivel transnacional, ya que esto incrementa el capital simbólico y añade prestigio tanto a los contrayentes como a sus familias.

Todas estas interrelaciones fueron tan evidentes desde el comienzo del trabajo de campo que, a pesar de que el proyecto inicial planteaba realizar la tesis doctoral sobre la comunidad sikh de Barcelona y llevar a cabo todo el trabajo de campo allí, pronto tuve que reformular la estrategia de investigación y virar hacia la etnografía multisituada.

\section{Etnografías multi-situadas y multi-focalizadas}

Cuando consideramos nuestros trabajos etnográficos no podemos obviar que el mundo de hoy se caracteriza por la desterritorialización y por la saturación de interacciones de múltiples órdenes e intensidades -algo que Grahame Thompson (1999) concretaba en el concepto de glocalización- y por los flujos culturales globales señalados por Arjun Appadurai (2001). Es, así, cada vez más incoherente -además de complicado- abordar cuestiones locales sin ponerlas en relación con las interconexiones que inevitablemente ocurren a nivel transnacional cuando no a nivel global. Más aún cuando trabajamos sobre migraciones o diásporas. Pues si consideramos como entidades discretas a comunidades migradas o diaspóricas, que suelen tener consideración de minorías étnicas o religiosas en los contextos de acogida, estaremos obviando las conexiones, interrelaciones y matices que forman parte de sus prácticas cotidianas; y que, de hecho, estas comunidades forman parte de grupos más amplios, en muchos casos diaspóricos y transnacionales. Y si no tenemos en cuenta estas cuestiones en nuestras observaciones y análisis, estaremos obviando una realidad más compleja y caleidoscópica de la que nuestros sujetos de estudio son parte y partícipes. Es, por tanto, sólo teniendo en consideración esta conexión fáctica e indubitable entre lo local y lo global que podremos incorporar en nuestro análisis muchos elementos que forman parte de una realidad mucho más interrelacionada y holística de lo que en un principio pudiera parecer. En este punto es además relevante considerar a Ghassan Hage (2005), quien mantiene que si consideramos a nuestro grupo de estudio como una familia o comunidad transnacional o global, no deberíamos considerar a sus miembros como sujetos pertenecientes a diferentes lugares o localizaciones, sino al mismo espacio, aunque este sea disperso. Es, por tanto, en este sentido, que debemos atender la necesidad de llevar a cabo etnografías que trasciendan los límites locales, y que puedan dar cuenta de la existencia y la singularidad que los flujos -como señalaba Appadurai (op. cit.) adquieren para el conocimiento y comprensión de nuestros grupos de estudio o el objeto de nuestras investigaciones antropológicas.

Si bien George Marcus (1995) señalaba dos formas de llevar a cabo las etnografías multisituadas, "follow the people" y "follow the thing", en mi caso definitivamente seguí a los sikhs de Barcelona hasta el Panyab y Delhi. Asimismo, también siguiendo las redes y

una parte de los ingresos que se demandan desde el centro de gobierno de los sikhs.

9 Un ejemplo local sería la emisora Sants3Ràdio, en el dial 103.2 FM, que durante mucho tiempo ha estado emitiendo los sábados entre las 21 y las $22 \mathrm{~h}$. un programa llamado "Un pont cap al Punjab". Asimismo, en las emisoras de radio panyabíes se emite con frecuencia información sobre los distintos acontecimientos y celebraciones que se suceden en diferentes lugares de la diáspora. 
conexiones que ellos tenían establecidas me trasladé a hacer trabajo de campo a Southall (Londres) para trabajar con amigos y familiares de sikhs establecidos en Barcelona.

\section{Diferentes contextos, distintas estrategias}

Cuando una comunidad llega y se establece en un nuevo contexto -como lo es Barcelona para los sikhs- y especialmente si es un lugar donde existe un desconocimiento generalizado sobre su cultura y/o religión seleccionan determinados rasgos distintivos que serán los que les caractericen en tanto que grupo étnico discreto. Siguiendo a Barth (1976), serán rasgos distintivos seleccionados como significativos por sus propios actores desde los que construir y recrear una representación social determinada a partir de sus experiencias compartidas. Esta creación identitaria formaría parte de la interacción y la negociación llevada a cabo entre el propio grupo y los otros grupos con los que interactúa. Así, por parte del propio grupo se elaboran y utilizan determinados símbolos culturales que se convertirán en las señas identitarias del mismo en el lugar y que tendrán la capacidad de mostrarlo como un grupo discreto y diferenciado frente a aquellos otros grupos con los que coexiste o se relaciona. Se trataría, de este modo, de construir una representación social determinada de la propia comunidad que, en constante (re)elaboración, identifica al grupo étnico en tanto que tal a partir de los elementos que el propio grupo considera significativos frente a los otros. Autoadscripción, identificación en base a unas características diferenciadoras seleccionadas por el propio grupo y otredad -ya que pese a ser colectivos discretos éstos no viven aislados ni por tanto ajenos a los grupos que les rodean-, serían la base constitutiva del grupo étnico diferenciado, que regula y limita la interacción y articula el contacto con los otros grupos a fin de mantenerse como tal.

En el caso de Barcelona, los sikhs refieren especialmente a los y las paquistaníes de forma específica y a los musulmanes en general como sus otros. También de forma muy particular las mujeres occidentales son consideradas en términos de otredad especialmente negativa desde que son un ejemplo no deseable para la construcción de la representación identitaria de las mujeres de la India. En este sentido, señalan a las mujeres occidentales como modelo que las mujeres sikhs no deben seguir, ya que son consideradas básicamente impuras, promiscuas y un mal ejemplo en general.

Sin embargo, los sikhs en Londres están mucho más preocupados en la construcción su auto-representación en términos políticos. Esta auto-representación identitaria se construye en base a la idea de una comunidad históricamente perseguida y tradicionalmente oprimida por el gobierno central de la India. Constantemente muestran y refieren a sus miembros como mártires y sufridores del desconocimiento e intolerancia hacia sus símbolos religiosos. En cambio, debido a su reciente asentamiento en Barcelona, la construcción de su representación en estos términos políticos no se da - al menos de momento. Pero si consideramos la larga trayectoria diaspórica de esta comunidad, estos ejemplos señalados nos dan suficiente información sobre las diferentes estrategias en base a la construcción de su representación que son llevadas a cabo por los sikhs en los distintos lugares de asentamiento. Y dentro de este planteamiento que refiere a que diferentes contextos requieren de distintas estrategias para el establecimiento y adaptación a los nuevos contextos, podemos encontrar a menudo cómo esas estrategias pasan por la alianza con diferentes grupos. Por ejemplo, si tenemos en cuenta que los panyabis son un grupo fuertemente endogámico, el trabajo de Karen Isaksen Leonard $(1992,1997)$ muestra cómo los matrimonios mixtos entre hombres panyabis y mujeres mexicanas se establecieron como una pauta generalizada en California debido a las limitaciones en las políticas de frontera que hacían imposible a los sikhs casarse con mujeres provenientes de la India. De una forma similar, en Barcelona podemos encontrar varones sikhs casados con mujeres barcelonesas (obviando su "otredad") para regularizar su situación y tener acceso a las redes locales. Por lo que pude observar 
durante el trabajo de campo, después de cierto periodo de tiempo se divorcian y vuelven a casarse, en esta segunda ocasión con mujeres hindúes ${ }^{10}$. También otra estrategia que me trasladaron mis propios informantes pasa por llevar a cabo matrimonios concertados con mujeres portuguesas o polacas a cambio de cierta cantidad de dinero para poder regularizar su situación en el país y poder moverse libremente por el territorio comunitario. En esta misma línea de estrategias, si bien mi sujeto de estudio eran los y las sikhs inmigrados desde la India, tuve necesariamente que llevar a cabo trabajo de campo entre los gora sikhs (hombres y mujeres occidentales que se siguen una especie de "sikhismo diferenciado u occidentalizado") dado que ellos eran el único contacto que las instituciones que trabajan con comunidades religiosas en catalunya tenían con los sikhs de la India. A pesar de que los gora sikhs y los sikhs de la India no tienen mucha relación -más bien se miran unos a otros con mutua desconfianza o recelo-, cuando los sikhs de la India comenzaron a asentarse en Barcelona recurrieron a los gora sikhs como grupo de contacto y como intermediarios con la población y las administraciones locales.

Todas estas situaciones señaladas hasta ahora llevan necesariamente a plantearse sobre la necesidad de llevar a cabo etnografías multi-focalizadas, en el sentido de que no podemos centrar ni limitar nuestra mirada de forma exclusiva hacia nuestro grupo sujeto de estudio. Pues su realidad son ellos y los grupos con los que en alguna u otra medida interaccionan, o no.

\section{Descubriendo a los gora sikhs}

Si bien como señalaba más arriba mis sujetos de estudio eran aquellos sikhs inmigrados, paradójicamente, cuando comenzaba la investigación y contacté con las entidades que trabajan con los diferentes grupos religiosos en Barcelona, me pusieron en contacto con el representante y portavoz del Consejo Sikh de Cataluña, un barcelonés de origen y aspecto occidental, de entre 45 y 50 años, de piel más blanca que la mía. Si bien no pudo -y creo que ni quiso- facilitarme mucha información sobre los sikhs de origen hindú, sí que comenzó el efecto "bola de nieve" que me llevaría hasta una joven sikh de origen barcelonés cuyo novio era sikh de origen hindú. Ella, sin duda, se convirtió en una buena informante y la mejor portera que podría encontrar. No obstante, hasta poder llegar a cierta integración en la comunidad sikh de origen hindú de Barcelona tuve que documentarme y llevar a cabo trabajo de campo entre estos sikhs de origen occidental y sobre los que ya han tratado autores como Cole (2004), Cole \& Singh Sambhi (2006) y Kaur Takhar (2005), entre otros. Conocer sobre los gora sikhs sería fundamental para no confundir las bases ideológicas y prácticas de un grupo con otro y no desvirtuar la idiosincrasia de mi grupo sujeto de estudio.

Los gora sikhs serían practicantes de lo que yo defino como un "sikhismo occidentalizado" y de los que pude encontrar varios miembros en Barcelona (algunos nativos, pero también otros provenientes de lugares como el Reino Unido y que habían llegado aquí después de pasar por lugares como Estados Unidos y América Latina). Asimismo, el trabajo de campo puso de manifiesto que los gora sikhs se encuentran también en otras ciudades del Estado Español. Con la idea de "sikhismo occidental" o "sikhismo occidentalizado trato de referirme a la práctica del sikhismo llevado a cabo por occidentales que resulta de la reformulación de algunos principios y prácticas del sikhismo tradicional adecuándolo así a las necesidades y a una ideología de la religión que necesariamente ha de responder en mayor medida a las necesidades y valores occidentales, evitando así los conflictos y los choques culturales que surgen con el sikhismo más tradicional. Es decir, se trata de personas de origen occidental, nacidas y socializadas en occidente, que practican una suerte de sikhismo diferenciado de los sikhs de origen hindú. Se trataría, de este modo, de dos grupos distintos que tienen en común algunos preceptos y prácticas del sikhismo pero que en principio no

10 Aunque también he podido conocer alguna situación de poliginia donde conviven el marido, la mujer barcelonesa y sus descendientes con la mujer de la India y sus hijos. 
se mezclan, permaneciendo como grupos diferentes que se ven y asumen entre ellos como absolutamente dispares. Así, los occidentales que practican este sikhismo -diferente, como digo, en algunos aspectos del sikhismo original de la India- reformulan aquellas ideas y pautas que no convergen con la ideología occidental, generando así una suerte de sikhismo diferenciado del primero que, más adecuado con los cánones de pensamiento y las pautas de actuación generales de occidente, sirve a sus practicantes para encontrar y desarrollar una suerte de misticismo o espiritualidad que les ofrece una respuesta alternativa a ciertas inquietudes y necesidades que en su propia cultura no logran solventar. Así, este "sikhismo occidental" o "sikhismo occidentalizado" resultaría una amalgama entre la religión sikh tradicional y ciertos valores de occidente que, en conjunción, ofrece a muchos occidentales una propuesta y la práctica de una creencia mística y religiosa que no entra en conflicto con sus propias prácticas, sus sistemas de pensamiento y sus valores.

Así, el concepto de "sikhismo occidental" o "sikhismo occidentalizado" resulta útil para denominar ese sikhismo mutado o modificado que se da y practica en los países occidentales y que comparte una serie de prácticas y la ideología de base del sikhismo tradicional, como la creencia en el Adih Granth o Gurú Granth Sahib ${ }^{11}$ y el resto de gurús, la práctica del sewa $a^{12}$, la no ingestión de carne animal, el rezo diario de oraciones de los mismos textos sagrados, el rito de iniciación a la $\mathrm{khalsa}^{13}$, etc. No obstante, también se diferencia de éste en aspectos tan fundamentales como su asociación a la práctica del kundalini yoga, el papel y el lugar que otorga a la mujer y su completa inserción en las prácticas mercantilistas del mundo occidental.

Esta particular forma de sikhismo comienza a calar entre los occidentales en 1969, cuando un sikh panyabi y profesor espiritual llamado Harbhajan Singh Puri, más conocido como Yogui Bhajan, comienza a enseñar kundalini yoga ${ }^{14}$ en Estados Unidos, donde una serie de estudiantes empiezan a sentirse atraídos por su modalidad de vida, que combinaba la meditación basada en las escrituras sikhs, una visión igualitaria de las personas y el compromiso personal hacia las mismas, la iniciación a la khalsa y una rígida disciplina que incluía la alimentación vegetariana y la prohibición de tabaco, alcohol o cualquier otro intoxicante del cuerpo, así como de la práctica del sexo fuera del matrimonio. Sería entonces a finales de ese mismo año cuando los primeros "convertidos" tomarían el amrit ${ }^{15}$. Según Cole \& Singh Sambhi (2006: 186), para el año 1975 su número se estimaba en 250.000 personas participantes y más de 110 centros establecidos.

A los sikhs occidentales se les conoce como gora sikhs -sikhs blancos- y se caracterizan principalmente por combinar el Sikh Dharma -el modo de vida sikh- con la práctica del kundalini yoga. Pese a que el número de sikhs occidentales ha ido creciendo y extendiéndose por gran parte de los países del mundo occidental, que tras la ceremonia de la khalsa son perseverantes portadores de las $5 K s^{16}$, que educan a sus hijos en el Sikh Dharma, que también consideran Amritsar ${ }^{17}$ como su centro religioso más importante, que viajan frecuente-

$11 \mathrm{Su}$ Libro Sagrado, al que atribuyen determinadas cualidades humanas y que es considerado como su último y definitivo gurú.

12 Realización de trabajos o aportaciones voluntarias para la comunidad.

13 Hermandad con cualidades guerreras que representa a los puros y en los que se materializan los valores e ideales del sikhismo.

14 Modalidad de yoga que va estrechamente ligada a ciertas técnicas de respiración.

$15 \mathrm{O}$ agua sagrada que forma parte del rito de iniciación a la khalsa.

16 Cinco elementos que simbolizan determinados valores religiosos y que se incorporan al cuerpo del iniciado tras el rito del amrit y a partir del que pasarán a formar parte del propio cuerpo. Estos son Kesh, el cabello sin cortar; Kangā, un peine de madera; Kirpan, una daga; Kachhera, una especie de calzones; y Karā, una pulsera de acero.

17 Lugar donde se ubica el Golden Temple, el templo más sagrado de los sikhs y que podríamos considerar como el equivalente a La Meca para los musulmanes. 
mente al Panyab y que envían a sus hijos allí a formarse y a estudiar en colegios sikhs, los sikhs de la India no les consideran como a iguales, y no les incluyen dentro de la panth -la comunidad sikh en su sentido más amplio- . A pesar de ello, los sikhs occidentales sí que se consideran como sikhs en toda regla, y en ningún caso se definen como seguidores de un grupo o secta particular o diferenciada -como sí los han definido algunos de mis informantes en la India, no necesariamente sikhs- sino como los auténticos sikhs.

Existen, no obstante, una serie de diferencias que crean una distancia esencial entre unos y otros. Así, podríamos destacar: en primer lugar, la base fundamental del sikhismo tradicional es la creencia en Akal Purah, un dios inmanente e inefable que se encuentra en todas las personas y en todas las cosas; en los transmisores de su palabra, diez gurús, nueve de ellos humanos y el último -y en sus palabras el "definitivo gurú"-, el Gurú Granth Sahib o Adih Granth (su libro sagrado). Aunque el rezo es individual, para el mantenimiento y la contemplación absoluta de la fe es fundamental la sangat o congregación (es decir, la vinculación a la comunidad).

En cambio, para los gora sikhs, el especial énfasis estaría en la práctica del kundalini yoga, que se personifica por la imagen de una serpiente enrollada, conocida como sakti (energía) que permanece aletargada o latente en la base de la espina dorsal de las personas. El objetivo del kundalini yoga no es otro que es despertar a este sakti que se desplazaría hasta los seis cakras o nervios centrales hasta alcanzar la coronilla de la cabeza, trayendo la iluminación espiritual y pudiendo llevar a la liberación espiritual. Esencial para ello es el pranayama, esto es, técnicas de respiración que ayudan a despertar el kundalini que permanece en estado de letargo en la base de la espina dorsal. Esto es algo absolutamente ajeno a los sikhs de origen hindú, para quienes contradiría el gurbani (las composiciones de los gurús que aparecen en el Adih Granth, donde se establece que la realización con Dios no viene a través de las técnicas respiratorias de los yogis $)^{18}$. De tal modo que la idea de espiritualidad y el énfasis en el bienestar espiritual está absolutamente presente en la ideología gora sikh, como rezaba en una reconocida frase de su líder Yogi Bhajan: "We are not human beings having a spiritual experience. We are spiritual beings having a human experience" (citado en Kaur Takhar, 2005: 160), frase que además sirve de reclamo publicitario habitual en las empresas relacionadas con este colectivo. A pesar de que los gora sikhs reconocen que la práctica del kundalini yoga realmente no está aceptada en el Guru Granth Sahib, manifiestan que su orientación es más saludable que la de la panyabi panth o comunidad.

Otra diferencia fundamental reside en la concepción que los gora sikhs tienen acerca del cuerpo. Para los sikhs de origen hindú el cuerpo sería esencialmente el contenedor del alma $(\bar{a} t m \bar{a})$, y esta es considerada parte de dios y que, aunque esencialmente diferente de este, comparte sus atributos -y por ello es inmortal- dado que el alma proviene de dios. Sería así como la divinidad está presente en cada persona a través del alma, que comparte parte de sus cualidades. En cambio, para los gora sikhs, como señala Kaur Takhar (2005: 166) y de acuerdo con el Sikh Dharma of the Western Hemisphere, bajo el cuerpo físico se encuentran diez cuerpos adicionales:

El cuerpo-alma: referiría al cuerpo eterno, descrito como la luz divina que vive en el corazón de las personas y sería similar a la concepción vista más arriba de un alma eterna vinculada a la deidad.

Tres "cuerpos mentales" (mental bodies) que serían los tres aspectos primordiales de la mente:

2.1. La mente negativa o negative mind: la que nos advierte contra los peligros

18 No obstante, sí se dan entre algunos sikhs de origen hindú "desviados" de las creencias más tradicionales -aunque son casos muy excepcionales- que tras mucho tiempo asentados en occidente se han acercado a la práctica del kundalini yoga, casos que sólo he conocido en Southall, Londres. 


\subsection{La mente positiva o positive mind: la inspiración}

2.3. La mente neutra o neutral mind: el conocimiento intuitivo basado en las dos mentes anteriores.

El cuerpo físico: un templo para que Dios viva en él.

El cuerpo pránico: controla la respiración y accede al prana, la energía vital del universo. Este cuerpo se fortalece mediante el pranayama, las técnicas respiratorias.

El cuerpo de arco o arc body: es la energía espiritual, a menudo llamado "halo".

El cuerpo áurico o auric body: el aura, la energía electromagnética de cada individuo.

El cuerpo sutil o sublte body: nos permite entender las situaciones que nos rodean.

El cuerpo radiante o radiant body: el coraje y carisma del individuo.

Diez cuerpos que estarían interconectados, de los que a la muerte sólo sobrevivirían el cuerpo alma y el cuerpo sutil que permanecerían en el siguiente reino de la existencia, ya sea el renacimiento o el $m u k t i^{19}$.

En cuanto a la imagen personal si bien los sikhs de la India en gran medida utilizan su atuendo personal para mostrar y conformar su arraigo identitario (Santos-Fraile, 2016), los sikhs occidentales visten de riguroso e impoluto blanco. Sucede también que, a diferencia de los sikhs de la India, la mayoría de los sikhs occidentales son kesdhari ${ }^{20}$ cuando no amritdhari si han tomado el amrit y, por tanto, adoptan y siguen con rigor el Sikh Rehat Maryada y exhiben una estética rigorista sikh en términos de la imagen o apariencia que la khalsa exige. Llevan la kara -la pulsera- y tanto los hombres como las mujeres portan turbante -normalmente también en blanco-, que es muy minoritariamente utilizado por las mujeres sikhs de India ${ }^{21}$.

Asimismo, como decíamos, les caracteriza su vinculación con el kundalini yoga -que a su vez asocian con el ideal de una vida sana y saludable, así como con un vegetarianismo más estricto que el que por norma general practican los sikhs tradicionales-.

Ocurre, por otra parte, que la construcción identitaria que como sikhs tienen unos y otros dista mucho de ser semejante. Unas mismas premisas ideológicas o religiosas no conforman unas características identitarias comunes -y aquí los procesos de enculturación tienen mucho que decir-. Tanto es así que ambos grupos tratan de diferenciarse tanto en su atuendo personal como en sus prácticas y rituales cotidianos. Un ejemplo bastante significativo al respecto puede ser la forma de saludo: mientras que los sikhs de la India se saludan uniendo las palmas de las manos y utilizando la frase "Sat Siri Akal" -que podría traducirse, según la fuente, por "la verdad se haga", o por "la gran verdad inmortal"-, los sikhs occidentales, con el mismo gesto, dirán "Sat Nam" -"en el nombre de la verdad", o también utilizado para referirse a Dios-, aunque ambos recurran al "Guaje Guru Ji Ka Khalsa, Guaje Guru Ji Ki Fateh" -"la victoria de la khalsa, la victoria del Señor", o "los puros pertenecen a Dios y toda la victoria va hacia Dios"-, para dar mayor énfasis al saludo o mostrar su estrecha vinculación con el sikhismo y su ideario.

Al tiempo que los sikhs occidentales se consideran a sí mismos verdaderos sikhs -aunque como hemos visto los sikhs de la India no estén en absoluto de acuerdo con esta apreciación-, piensan que los sikhs tradicionales han ido perdiendo parte de sus valores, y que ellos son los encargados de hacerlos resurgir. De este modo, afirman que son más serios y

19 Mukti generalmente se traduce por liberación, y en sintonía con otras tradiciones de la India puede alcanzarse en vida. En el sikhismo esta emancipación consistiría en llegar a ser uno con la divinidad (Pániker, 2007: 56).

20 Aquellos que no cortan ningún pelo de su cuerpo y utilizan turbante.

21 Normalmente el color blanco es el que visten las viudas y el que se usa cuando se asiste a un funeral. 
estrictos en su práctica del sikhismo que los sikhs tradicionales, que se han relajado en sus prácticas y en su fervor religioso. Así, los sikhs occidentales son entusiastas kesdharis y portadores de las $5 K s$, practicas utilizadas incluso para los más pequeños. No obstante, si hay algo que realmente diferencia y distancia a unos sikhs y otros, tiene que ver con el bagaje cultural que unos y otros arrastran. La identidad de un sikh de la India es indisociable de su origen panyabi, y los elementos que les constituyen en tanto que sikhs y en tanto que panyabis son difícilmente separables y diferenciables. En esta línea, para Opinderjit Kaur Takhar (2005: 173) hay un elemento fundamental en la cultura y en la etnicidad panyabi, la noción de $i z z a t^{22}$, el honor, que es fundamental para la familia y la comunidad, y que está absolutamente presente en las normas básicas de la sociedad; por el contrario, parece que nada más ajeno que esta idea en la ideología moral de los sikhs occidentales. Como señala esta autora, citando a Dusenbery,

"the moral sensitivities of Gora Sikhs and Punjabí Sikhs (and, in this regard, particularly those of the dominant Jat Sikhs) not only differ but also differ in culturally specific ways...the notion of "izzat", apparently so central to Jat Sikh "moral affect", is not shared by Gora Sikhs...this difference in moral affect, I argue, enters into the active estrangement of Gora Sikhs and Jat Sikhs and their (mis)apprehension of one another" (Cit. en Kaur Takhar, 2005: 173).

Cabe señalar también que el lugar que ocupa la mujer dentro de la sociedad -con un rol pasivo entre los sikhs de la India- es bien distinto al que desempeña la mujer entre los sikhs occidentales. Estas últimas ejercen un papel activo al que no están dispuestas a renunciar, son practicantes y profesoras de kundalini yoga, ejercen cargos ministeriales dentro de la organización y, sobre todo, son bastante críticas y revulsivas con el rol que se atribuye a la mujer sikh hindú. Otra de las características más importantes que les diferencia consiste en que los sikhs de la India normalmente han nacido y se han socializado dentro de la comunidad sikh, donde la adscripción religiosa viene normalmente por filiación patrilineal, por lo que su adscripción no responde tanto a una elección tomada en un determinado momento de la vida, sino que se considera algo dado. En cambio, la mayoría de los sikhs occidentales son personas convertidas al sikhismo (excepto los hijos nacidos dentro de un matrimonio entre sikhs occidentales), lo que puede ser la principal razón de su purismo religioso. Así, los sikhs occidentales conocen y se aproximan al sikhismo a través de la práctica del kundalini yoga y habitualmente después de haber pasado por importantes o sucesivas crisis vitales. Cuando pregunto a los informantes occidentales qué es lo que más les atrae del sikhismo o qué les hizo hacerse sikhs, sus respuestas tienen que ver con la búsqueda de un sentido, de algo que no pudieron hallar de otras formas o en otros lugares; asimismo, señalan el hecho de que el mensaje de Gurú Nanak (el primer y más importante gurú) sea un mensaje universal, además del hecho de poder vivir la espiritualidad en familia -ya que los ministerios sikhs no se oponen a una vida marital-. Parece que terminan hallando en el sikhismo lo que ellos definen como una forma de vida, que llena de mantras y pequeñas prácticas todo su universo cotidiano: ya sean actividades como el montarse en un coche, coger un tren o un metro, ingerir algún tipo de alimento, o simplemente caminar por la calle; acciones cotidianas que son reflexionadas y ritualizadas, que terminan imbuidas de determinada corporalidad y ejercicios de respiración, como muestra el siguiente mantra que me enseñó Kamaljit, y que hay que recitar antes de conducir o viajar, ya que nos mantendrá protegidos de accidentes: tras frotar las palmas de las manos, y manteniéndolas unidas, recitaremos:

Ong Namo Guru Dev Namo (3 veces) [esto también se utiliza cuando se hace

22 También podría traducirse por prestigio. 


\author{
kundalini yoga] \\ Ade Gurah Eh Nah meh! \\ Jugad Gureh Eh Nah meh! \\ Sat Gureh Eh Nah meh! \\ Sri Guru Dev En Nah meh! (repetirlo 3 veces)
}

Otra de las grandes características de los sikhs occidentales -a mi juicio quizás la más importante-, y que refleja la absoluta inmersión de esta creencia y su práctica -como decía siempre asociada al kundalini yoga- en el mundo occidental y que, además, la aleja sustancialmente de los sikhs de la India, es que se conforman en una serie de organizaciones administrativas y mercantiles supranacionales y jerárquicas que controlan y gestiona su dispersión y comercialización -así como la de sus productos- por los distintos países; así como la existencia y la gestión de toda una red de empresas internacionales. Su mayor escala en esta organización sería el movimiento denominado Sikh Dharma of the Western Hemisphere, que, aunque en relación con el kundalini yoga, pone sobre todo su énfasis en el Sikh Dharma; y la organización $3 H O$ (Healthy, Holy and Happy Organization), que sí tiene como su nexo central el kundalini yoga. Ambas están creadas por Yoghi Bhajan -considerado el jefe religioso y la máxima autoridad administrativa-, y la gran mayoría de las y los sikhs occidentales están vinculados con ellas. También está el Khalsa International Business and Trading, grupo de empresas (concretamente distribuidas en 14 corporaciones), cuyas actividades van desde el desarrollo de software informático y tés de hierbas hasta empresas de seguridad privada, que, según señala Opinderjit Kaur Takhar,

the list of companies (...) referred to as 'KIIT' is 'a group of companies that exist to serve, heal and uplift humanity through quality products and services ${ }^{23}$. The KIIT group includes such companies as the book- ordering service called 'Ancient Healing Ways Catalogue' which trades in all kinds of material with regard to the $3 \mathrm{HO}$ and Sikh Dharma of the Western Hemisphere. Other companies include 'Golden Temple Enterprises' from where literature may be obtained, 'Yogi Tea', which markets caffeine-free herbal tea, as well as 'Sunshine Products' which make available natural body and minds stimulants (Kaur Takhar, 2005: 160).

También existen cadenas de restaurantes conocidas como "Golden Temple Consciousness Cookeries" en Estados Unidos y Europa, que sirven exclusivamente cocina vegetariana y que igualmente forman parte de esas estructuras organizadas de los sikhs occidentales.

Además, con el inicio del Khalsa Council en 1974, se designan una serie de ministros -con la denominación de Singh Sahib para los varones, y Sardarni Sahiba para las mujerespara coordinar y gestionar las necesidades que surgen con el creciente número de alumnos que forman parte del Western Hemisphere. Estos "ministros" -que son considerados como sirvientes de la comunidad- serían los encargados del bienestar de la sangat -la congregación religiosa- en sus respectivas áreas que están delimitadas geográficamente. Ya en su día, Yogui Bhajan se encargó de distribuir alumnos suyos ya convertidos en "maestros" por diferentes países occidentales con la misión de crear nuevas escuelas de kundalini yoga y difundir así la práctica del sikhismo (proselitismo que no se da entre los sikhs de origen hindú). Por otra parte, los centros establecidos por Yogui Bhajan, en los que se llevan a cabo las prácticas sikhs entre las que no faltan los $\operatorname{ardas}^{24}$ como oración fundamental del sikhismo, son también conocidos como ashrams, lo cual sería otra forma de distinción -por su denominación y gestión- con respecto a los gurdwaras, lo que viene de nuevo a reafirmar cierta separación voluntaria con respecto al sikhismo tradicional.

Todo ello refleja cómo el sikhismo occidental está absolutamente insertado en el modo

23 KIIT Mission Statements, Los Angeles: 3HO Foundation.

24 Plegaria fundamental que se recita cuando concluyen muchas ceremonias sikhs. 
de vida de occidente y también en sus prácticas capitalistas dentro estructuras jerárquicas y cerradas. Un último y más cercano ejemplo ilustrativo de ello es que para poder impartir clases de kundalini yoga en nuestro país - aunque se posea la cualificación como profesorhay que estar registrado en la Asociación Española de Kundalini Yoga (AEKY), órgano a su vez representante de la Asociación Internacional de Kundalini Yoga (IKYTA) y del Instituto Kundalini de Investigación (KRI) en España. Este registro ha de renovarse anualmente mediante el pago de la cuota correspondiente, sin la cual no es posible ejercer ${ }^{25}$, de modo que sólo se acredita a una persona como profesor o profesora de kundalini yoga mientras esté pagando las cuotas, y en el momento que deje de pagarlas, le retirará tal acreditación. Pero además de ello, en las escuelas de kundalini yoga se dispone de toda una serie de productos a la venta -también a través de Internet en el apartado de tienda on-line de sus webs- como son el Yogui Tea - una clase de té para la práctica y los practicantes de kundalini yoga libre de cafeína o estimulantes-, CDs de música New Age, DVDs, manuales y otros libros relacionados con el kundalini yoga y los mantras del sikhismo, la relajación y otras terapias, el misticismo y formas de encuentro y superación personal, además de otros productos útiles para la meditación y la práctica del yoga. Todo ello disponible para la compra de los interesados.

En fin, la visible mercantilización del sikhismo occidental asociada a la práctica del kundalini yoga desvela un trasfondo económico que se muestra como el aspecto más destacado de este grupo. Hasta donde ha llegado el alcance de esta investigación esto se observa como una cualidad muy diferenciada del modo de organización y gestión de la comunidad de los sikhs de la India, en la cual este carácter económico no aparece -al menos de momento- de forma tan evidente.

\section{Consideraciones finales}

Conocer sobre los gora sikhs fue sin duda condición indispensable para poder acercarme, introducirme y aprehender sobre los sikhs venidos de la India. Aprender las diferencias fundamentales que a nivel identitario construyen y en las que se basan uno y otro grupo fue altamente necesario para poder discernir la idiosincrasia de cada grupo y, especialmente, las estrategias adaptativas de ambos. Por un lado, de los sikhs de la India para adentrarse y establecerse exitosamente en un nuevo contexto (en este caso en Barcelona) y, por otro lado, de los gora sikhs para seleccionar y reformular determinados elementos de la ideología religiosa y adecuarlos e introducirlos, por un lado, en las prácticas mercantilistas occidentales $\mathrm{y}$, por otro, para cubrir las necesidades espirituales o carencias de un modo de vida que determinadas personas consideran como incompleto. Si bien al inicio de la investigación ni siquiera conocía la existencia de los gora sikhs, estos devinieron sujetos fundamentales para el avance del trabajo.

Por otro lado, otra cuestión que quisiera incorporar en la reflexión sobre las distancias que a veces se dan entre el planteamiento de la investigación y su decurso, es la cuestión de género. Durante mi experiencia de trabajo de campo, ser una mujer trabajando en una comunidad fuertemente patriarcal como lo es la comunidad panyabi, hacía prácticamente imposible mi acceso al mundo de los hombres sikhs. Afortunadamente, pude solventar esta dificultad persuadiendo al que por entonces era mi compañero sentimental -que no es antro-

25 Según especifica literalmente en su página web la Asociación de Kundalini Yoga, "Su objetivo es la certificación de profesores que hayan completado el programa oficial de KRI, el sostenimiento de un marco legal que asesore y proteja a los profesores, la difusión y preservación de las enseñanzas de Kundalini Yoga en nuestro país, además de la creación de dinámicas de interrelación entre los miembros y la comunidad de practicantes de Kundalini Yoga. Cuenta, además, con delegaciones en Catalunya, Madrid, Galicia, Comunidad Valenciana, así como en Andorra para canalizar, por lo que respecta a los socios de esas áreas, una mejor participación y apoyo. Actualmente está integrada por más de 800 socios". Recuperado el 13 de octubre de 2017 de: http://www.aeky.es/ 
pólogo- para que me acompañara a hacer trabajo de campo y realizara las entrevistas (con el guión y las instrucciones previas que yo le había facilitado antes) mientras yo tomaba notas, cual eficiente secretaria, manteniéndome siempre unos pasos por detrás, como las pautas culturales exigían.

Todas las situaciones expuestas a lo largo de este texto llevan necesariamente a reflexionar sobre una serie de dificultades y plantear interrogantes. Por un lado, los trabajos multi-situados han tratado de señalar las limitaciones de aquellas etnografías intensivamente focalizadas, tratando de resolver las deficiencias que en este sentido el contexto mundial impone. Pero, por otro lado, estos trabajos tampoco carecen de dificultades y cuestionamientos sobre su viabilidad real y sobre la satisfacción en los resultados obtenidos. También sobre la propia experiencia etnográfica. Refutaciones, ansiedades, dificultades para marcar los límites de la etnografía, así como la disminución de la efectividad del trabajo de campo, se han planteado como obstáculos o desventajas que acompañan este tipo de trabajo, como el propio Marcus señalara:

Among anthropologists, the move toward multi-sited ethnography might give rise to three sets of methodological anxieties: a concern about testing the limits of ethnography, a concern about attenuating the power of fieldwork, and a concern about the loss of the subaltern (1995: 82-83).

En este sentido, la imposibilidad de delimitar el campo de estudio a un área restringida, accesible y cognoscible produce una constante sensación de "descontrol" e ilimitación; así como la necesaria temporalidad de la estancia en cada una de las localizaciones del trabajo de campo también genera cierta ansiedad y sensaciones de insuficiencia con respecto al trabajo de campo, pues es constante la sensación de que nunca dedicas el tiempo suficiente a cada uno de los sitios y los grupos de estudio, y continuamente te cuestionas acerca de lo que estará ocurriendo en los sitios donde no estás o si lo que observas acontecería allí o de qué manera sucedería -circunstancias que por otro lado difícilmente se pueden conocer y comprobar precisamente por la necesidad de ceñirte a lo que ocurre o apremia en el lugar en que te encuentras y en los tiempos en que en ellos estás.

Asimismo, el cambio de localizaciones, así como la premura que la temporalidad requiere generan sentimientos a veces encontrados o de ambivalencia, ya que es condición necesaria de la etnografía multi-situada la renegociación constante del rol del/de la investigador/a en función del lugar y el contexto en el que se encuentra, algo que Marcus (op. Cit: 97-99) también ya señalaba. Pero además, no debemos olvidar que la etnografía necesariamente requiere implicación, dedicación y compromiso, una suerte de arraigo al campo y con los temas de estudio que ciertamente es difícil de mantener en diferentes contextos.

Teniendo todo ello en consideración podemos afirmar que los cambios, cada vez más acelerados en el mundo global, requieren del replanteamiento de la práctica etnográfica entendida como un trabajo en soledad respecto de una comunidad discreta, y no ya sólo cuando se trata de trabajos sobre migraciones o diásporas, sino sobre cualquier grupo o comunidad que inexorablemente permanece interconectada y afectada por los cambios en los flujos globales actuales y el resto de dinámicas de la globalización. La etnografía multisituada, que daría cuenta tanto de esas interrelaciones y conexiones, que además permite considerar a un mismo grupo de estudio como espacialmente diversificado, es una alternativa útil y eficaz que permite mostrar de forma más compleja la realidad de los individuos en la situación mundial actual. Asimismo, dejar de lado la idea del/la etnógrafo/a anacoreta que se enfrenta al campo en soledad malinowskiana, y plantear trabajos con equipos de profesionales que desarrollan su labor de forma simultánea en diferentes lugares puede ser una alternativa que dé resultados más completos y realistas sobre las experiencias compartidas de los sujetos y grupos culturales en este mundo globalizado que demanda etnografías más complejas, holísticas y caleidoscópicas. Sin obviar que un trabajo colaborativo o en grupo nos permitiría acercarnos con mayores garantías a la multi-focalidad que en gran medida 
requieren las etnografías en este mundo que como decimos está incuestionablemente interconectado. Ello nos permitiría, además, superar las dificultades derivadas de la cuestión de género y realizar etnografías más completas cuando los equipos de trabajo estuvieran compuestos por hombres y por mujeres profesionales de la antropología. Poner en práctica esta propuesta será la forma de ver su viabilidad a tenor del éxito de los resultados obtenidos.

\section{Bibliografía}

Anderson, B. (2000). Comunidades imaginadas. Reflexiones sobre el origen y la difusión del nacionalismo. Buenos Aires, Argentina: Fondo de cultura Económica.

Appadurai, A. (2001). La modernidad desbordada. Dimensiones culturales de la globalización. Buenos Aires, Argentina: Fondo de Cultura Económica.

Barth, F. (1976). Introduction. En Barth, F. (Comp.), Ethnic groups and boundaries: 9- 38. Long Grove, Illinois: Waveland Press.

Cole, O. W. (2004). Understanding Sikhism. Edinburgh, UK: Dunedin Academic Press.

Cole, O. W. and Singh Sambhi: (2006) [1995]. The Sikhs. Their Religious Beliefs and Practices. Brighton-Portland: Sussex Academic Press.

Godelier, M. (2010). Community, society, culture: three keys to understanding today's conflicted identities. Journal of the Royal Anthropological Institute 16 (1): 1-11. DOI: https://doi. org/10.1111/j.1467-9655.2009.01593.x

Hage, G. (2005). A not so multi-sited ethnography of a not so imagined community. Anthropological Theory, Vol. 5 (4): 463-475. DOI: https://doi.org/10.1177/1463499605059232

Hammersley, M. y Atkinson: (1994). Etnografía. Barcelona, España: Paidós.

Leonard, K. I. (1992). Making Ethnic Choices. California's Punjabi Mexican Americans, Philadelphia: Temple University Press.

Leonard, K. I (1997. The South Asian Americans. London, UK: Greenwood Press.

Lum, K. (2009). A minority within a minority: the Ravidassia Sikhs. Recuperado de http://www. sikhs-in-europe.org/index.php?option=com_docman\&task=doc_view\&gid=49\&tmpl=co mponent\&format $=$ raw \&Itemi $\mathrm{d}=60$

Lum, K. (2011). How caste works: Forging New Identities in a Punjabi Ex-Untouchable Community in Catalonia, Spain (Tesis doctoral). European University Institute, Florencia.

Malinowski, B. (1989). Diario de campo en Melanesia. Madrid, España: Júcar.

Marcus, G. (1995). Ethnography in/of the world system: The Emergence of multi-sited ethnography. Annual Review of Anthropology, 24, 95-117. DOI: https://doi.org/10.1146/annurev. an.24.100195.000523

Matringe, (2008). Les Sikhs: Histoire et tradition des "Lions du Panjab", Édition Albin Michel.

McGregor, R. S. (Ed.) (2004) [1993]. The Oxford Hindi-English Dictionary, Oxford; New York: Oxford University Press.

McLeod, W.H. (2004) [2000]. Exploring sikhism. Aspects of sikh identity, culture and thought. Oxford, UK: Oxford University Press.

McLeod, W.H. (2006) [1996]. Sikhs and sikhism. Oxford, UK: Oxford University Press.

Monier-Williams, M. (1996). Sanskrit-English dictionary: etymologically and philologically arranged. Godowila: Indica.

Oberoi, H. (1994). The construction of religious boundaries. Culture, identity and diversity in the Sikh tradition. Delhi, Oxford, New York: Oxford University Press.

Oberoi, H. (1997). Sikh fundamentalism. En Kaviraj, S. (Ed.). Politics in India. New Delhi: Oxford University Press.

Pániker, A. (2007). Los sikhs. Historia, identidad y religión. Barcelona, España: Kairós.

Portes, A. (2007). Migración y desarrollo: una revisión conceptual de la evidencia. Migración y Desarrollo: perspectivas desde el sur: 21-49.

Prat, J. (2007) [1997]. El estigma del extraño. Un ensayo antropológico sobre sectas religiosas. Barcelona, España: Ariel.

Pujol, O. (2005). Diccionari Sànscrit-Català. Barcelona, España: Enciclopèdica Catalana.

Santamaría, E. (Ed.) (2008). Retos epistemológicos de las migraciones transnacionales. Barcelona, España: Anthorpos. 
Santos-Fraile, S. (2007). La comunidad Sikh en Barcelona. Una aproximación etnográfica. (Trabajo de investigación de segundo año del programa de Doctorado (DEA) en Antropología Social y Cultural). Universitat de Barcelona, Barcelona.

Santos-Fraile, S. (2016). La comunidad sikh en Barcelona. Una aproximación etnográfica: Prácticas, negociación y transformaciones en el cuerpo y la corporalidad tras el proceso migratorio. (Tesis Doctoral). Universitat de Barcelona, Barcelona.

Sayad, A. (2006). L'immigration ou les paradoxes de l'altérité. Paris, France: Éditions Raisons d'Agir

Takhar, O. K. (2005). Sikh Identity. An exploration of groups among Sikhs. Aldershot, UK: Ashgate Publishing Company.

Thompson, G. (1999). Introducción: situar la globalización. Revista Internacional de Ciencias Sociales (UNESCO) 160: 1-17.

Vertovec, S. (2009). Transnationalism. London \& New York: Routledge. 